\title{
A model system for psychotherapy research data management
}

\author{
REX S. GREEN \\ Systems Research Unit, Eastern Pennsylvania Psychiatric Institute, Philadelphia, Pennsylvania 19129
}

and

C. CLIFFORD ATTKISSON

Department of Psychiatry, University of California, San Francisco, California 94143

\begin{abstract}
An on-line data management system was developed to provide optimal access to research data. The specific application involved collecting data on four outcome measures from patients receiving brief psychotherapy to ameliorate a stress response syndrome. The on-line data management system also was designed to be transported to other research settings; a variety of other applications of this system are suggested. The need for such a system, the elements that it comprises, its operation, and its availability also are covered.
\end{abstract}

The quality and timeliness of research endeavors often depend on how well the more mundane tasks are handled. Keeping track of the data is one such chore. While a few boxes of card data are readily managed, keeping track of the same respondents for up to 2 years, when each respondent is completing a multiinstrument assessment battery on several occasions throughout this period, requires considerable skill and effort.

Most research endeavors encounter problems, such as the following, that compound the difficulties of managing research data. For example, new information may spark new hypotheses, for which little or no data are being collected. Budget changes may require revisions of the research design that alter the volume or nature of the data being collected. Key personnel may leave, assume different duties, disperse to additional sites, and so forth. New measurement or statistical procedures may be developed, requiring rescoring of the data or further analyses. Deadlines draw near and faster turnaround on analyses becomes imperative. Numerous unplanned analyses emerge as crucial but cannot be performed for lack of time or money.

When quality research depends on systematic and

R. S. Green is Evaluation Research and Scaling Specialist, Systems Research Unit, Eastern Pennsylvania Psychiatric Institute, Philadelphia, Pennsylvania. C. C. Attkisson is Associate Professor of Psychology, Department of Psychiatry, University of California, San Francisco, California. Support for research and system development reported in this paper derived from a postdoctoral fellowship in the UCSF Clinical Innovation and Evaluation Training Program. Grant 5-T32-MH14609.02 (Dr. Green) and from a National Institutes of Health, Biomedical Research Support Award (United States Public Health Service Grant RR-05755). Reprint requests should be sent to C. C. Attkisson, Department of Psychiatry, University of California, San Francisco, California 94143. precise data handling, with rapid turnaround on analyses, behavioral research projects should develop an on-line system for managing their research data. An optimal on-line data management system (ODMS) should provide unrestricted and inexpensive access to reliable, relevant, and comprehensive data, at the push of a button. Report formats should enhance one's understanding of a set of results and be simple to modify when necessary. The organization of the data files and data elements should be clear, concise, and flexible. The research director should be able to obtain a brief report of the current status of all data at any time. Then, when modifications of the research design are made, the research director can better ensure that appropriate data will be available in a timely manner. Finally, a minimal amount of time and money should have to be diverted to creating the ODMS.

Two years ago, we obtained access to both the hardware and software for constructing an ODMS for a psychotherapy research team. However, utilizing the computing technology took considerably longer than anticipated. We also learned that the process of creating the ODMS is crucial. Thus, this report focuses on the process of creating an ODMS, as well as its design and operation. Hopefully, reporting our efforts will assist other researchers to construct more optimal ODMS for their settings.

First, the computing technology that supports the creation of an ODMS is described. Second, the highlights of the process of developing this ODMS are covered. Third, its design is presented, followed by more specific details about its structure and operation. Fifth, ways of borrowing from this ODMS are suggested. Finally, its form and availability are spelled out. 


\section{INTERACTIVE DATA MANAGEMENT TECHNOLOGY}

In this section, we describe the three basic elements of computing technology that supported the creation of our ODMS. The two software elements were a computing program for storing and retrieving data that allows its users to "speak in English" when addressing it and a "friendly" interactive operating system (IOS) for on-line computing. The third element was a computer sufficiently large and rapid to support on-line or real-time data processing. Thus, the ODMS consists of English-like statements, grouped into routines, that "tell" these two computer programs to either store or access the research data. These routines are executed so rapidly in realtime as to engage the user in a dialogue.

In 1968, a computer software vendor, Cincom Systems, began stressing the need for computer programs that manage files of data. Soon, the concept of a "data base" as a collection of interrelated data elements, stored so as to be independent of the computer programs written to manipulate them (Martin, 1976) was born. A computer program written to create and maintain a data base came to be called the data base management system (DBMS). (See Appendix for an explication and summary of acronyms.) DBMS proved especially useful to large businesses. Consequently, the 1970s ushered in the technology of DBMS (Martin, 1976, 1977). Differing programs abound, as indicated by two recent reviews of DBMS (Rosenberg, 1980; Stiefel, 1979). Also, an international symposium comparing 27 DBMS was held in June 1980 by the Institute for Science and Public Affairs.

The following list of typical features of a DBMS illustrates why the use of DBMS is now widespread. Most DBMS have: (1) highly organized and compressed storage of data in related files; (2) an Englishlike, nonprocedural language for storing and accessing the data, sometime with an English grammar structure; (3) controlled access through various security provisions; (4) batch and interactive modes of access for both data entry and retrieval; (5) linkage features that enable user-written programs and system executive routines to operate on the data base; (6) data screening and editing options for data entry; (7) report generators with a variety of formatting, data selection, and data transformation options; (8) facilities for reading or creating external data files; (9) a means of linking different system files for a particular analysis or report; (10) aids for recovering from computer system failures during input; (11) speedy, on-line handling of the spontaneous access to a single record; (12) utility routines that assist the user in creating applications (e.g., file backup, file status reports, and saved procedures execution); (13) a data base dictionary that summarizes file and data element definitions; (14) special training courses, users' group programs, and a newsletter, to keep users informed of new features and system revisions; and (15) direct-dial, hotline support for user application problems.

Clearly, the interactive computing features of a DBMS support the creation of an ODMS for research data. The English-like commands for operating the report generator on-line can be used as follows. Several hypotheses can be formulated and tested on the same data in rapid sequence by designing and executing a series of report request routines to generate external files for each statistical analysis. Then, the user processes these files with a statistical package on-line, using an IOS. Turnaround time for such analyses depends primarily on how rapidly the researcher can interpret the results.

Creating an ODMS depended, too, on the availability of a powerful IOS. This software should perform in a friendly manner (i.e., "speak English"), activate many default options, unless the user specifically overrides them, and be forgiving of commonly occurring mistakes, such as misspelled commands or incorrect erasure of files. Further desirable features include: a nonprocedural language for creating executive routines to automate data handling, a text or file editor that can be operated via executive routines, file copying capabilities for merging selected lines and columns of different files, a powerful sort routine, and, of course, linkage to the DBMS.

On-line systems require more powerful and reliable computing hardware than batch systems, plus reliable data communications equipment. If the on-line system breaks down frequently, disengages too readily, or is overworked when access is needed, making response times unreasonable, the ODMS will not be able to perform optimally. Furthermore, the main-frame computer, or minicomputer, must perform its calculations rapidly enough to support on-line access. "Fast" is relative. A more powerful, but overworked, mainframe computer, or its data communications hardware, may not perform in real-time as rapidly as a minicomputer dedicated to one terminal.

\section{CREATING A PSYCHOTHERAPY RESEARCH ODMS}

Following Broskowski and Attkisson (in press), we organized this discussion of the development of our ODMS around the following major influences: the user's information needs, project constraints related to the organizations and people involved, and the specific computing technology utilized.

First, a word of caution: The computerization of an information system can become the Achilles' heel of needy organizations. While research endeavors are probably less steeped in tradition, more accepting of rapid change, and more willing to innovate than 
businesses or government service agencies, they will hardly be unaffected by the problems inherent in implementing an ODMS. The key principle to follow in designing and implementing an ODMS is to "work backward." Start with what is needed and step back to the present situation. Martin (1976), for one, maps out the road to the successful implementations of a DBMS, and he notes the likely pitfalls along the way. Broskowski and Attkisson (in press) step the beginner through the process of creating an information system for health and human service organizations. Johnson (1980) offers a practical guide for installing a computer system in a mental health setting. Other references of value to consult are: Attkisson, Hargreaves, Horowitz, and Sorensen (1978), Chapman (1977), Davis (1974), Enger (1976), Hargreaves, Attkisson, and Sorensen (1977), Krauss (1970), and Sidowski, Johnson, and Williams (1980).

From the viewpoint of the researcher, obtaining an optimal ODMS is best realized by discerning at the beginning what one's information needs are. In our setting, the psychotherapy research team was investigating brief treatment techniques for stress response syndromes. Data were accumulating, due to the longitudinal nature of the project. A recent influx of new funding prompted an expansion of the patient population. The "newness" of their techniques stimulated much hypothesis generating. The dearth of reliable and valid indicators of the impacts of psychotherapy mandated a multimeasure assessment approach (Waskow \& Parloff, 1974). Seemingly, there was much to gain by automating data entry and retrieval beyond batch accessing of SPSS (Nie, Hull, Jenkins, Steinbrenner, \& Bent, 1975).

The following information needs were identified through discussions with the psychotherapy research project director and his data processing staff: (1) Additional reports of patient status were to be created, circulated, and then filed as soon after a rating session as possible. (2) Four outcome assessments for all patients across all rating sessions were to be automatically stored together in an accessible manner (data were falling through the cracks of several data processing procedures). (3) Summary scores were to be computed from the raw data, following editing and checking, by forming sums of selected subsets of scale items, adjusting these subscale scores to handle missing values, and forming percentile and standardized scores. (4) The new system should handle frequent creation or revision of scales without reorganization of the files. (5) Rapid turnaround time from the report generator was needed to handle a variety of hypothesis-testing queries addressed to the data base at any time. (6) Easy linkage of the outcome data to other data stored in different forms on these same patients was desired (e.g., process note data, demographic information, interviewer's follow-up notes, etc.). (7) The design of the ODMS should support multiple input data streams, two of which were operative, with the addition of another being contemplated.

Along with the determination of the researcher's information needs, the constraints on the development of the system were appraised. Fiscal arrangements were discussed. Time lines were set in accordance with the availability of the systems programmer and research data processing staff. Since previously collected and processed data were to be entered into the data base, the status of those data had to be considered. Arrangements were discussed for realigning research data processing staff positions in order to better operate and maintain the ODMS.

Other constraints, more subtle by nature, gradually emerged. The psychotherapy research team evinced little interest in obtaining training in the use of the DBMS. Nor were staff members eager to convert more of their operation from batch to on-line data processing. As a result, the development of the system shifted more emphasis onto the automation of all data entry procedures and the creation of a filing structure for the data base that would be easily understood by the researchers. We began to consider alternative implementations of the ODMS. The design of the files was adjusted to serve similar research endeavors, especially psychometrics and evaluation research.

Of course, the research setting changed in a number of ways during the system development phase. Statistical software packages became more useful to the psychotherapy researchers, making this ODMS appear less of an improvement. Key support personnel on the team left. "Bugs" in the DBMS and new features incorporated in the IOS slowed development. Coordination with personnel developing related systems for the researchers waxed and waned. Such interferences invariably occur and must be dealt with in a timely manner.

With regard to the computer technology, the selection of the DBMS and IOS was determined by what the host university made available at their computing facility. The DBMS, which stimulated the creation of this ODMS, is called RAMIS II (Mathematica Products Group, 1977). Some idea of how RAMIS compares with other DBMS can be obtained from a recent interview article (Rosenberg, 1980). Our computing facility also performed an exacting product evaluation, contrasting eight DBMS, before acquiring RAMIS, the clear favorite (Karpinski, Note 1). The IOS will be described, following the discussion of our utilization of RAMIS.

RAMIS consists of computer programs and utility routines that store and retrieve data from hierarchical files, following the instructions from English-like 
routines the user devises. Hierarchical files conserve storage, speed access to selected records, and reduce data maintenance, because the recurring values of a data field, located in the higher levels of a hierarchy, need be stored only once. Pointers, which redirect a computer search operation to noncontiguous storage locations, are created by the DBMS to link data elements between levels of the hierarchy. Ideally, each unique data value is stored only once, and yet it is linked to all the other values that it was associated with in the records of the original sequential file. To accommodate those data relationships that are not hierarchical, RAMIS uses pointers to link different files. A "virtual" level is added to one file, in which only a description of the additional data is stored; data elements at the next higher level are designated as "keys"; the same key values are stored in another file, in which the additional data are stored; and pointers are created to link matching key values across files. The additional data can then be accessed as though they were part of the same record in one file. The file definitions, describing how and where all data are stored, are listed upon request. They succinctly describe each data field in each file, as well as the interfile linkages.

For example, a simple application might involve designing a two-level file of two variables, patients and their dates of therapy, and linking this file to a master file of other patient data. A RAMIS input routine, of, say, 6-10 lines, could be written to enter the card data in the new file, once the new file structure is entered in the data base and the master file structure is revised by adding the virtual level to the hierarchical file structure. Only the master file design requires modification, not the master file of data. RAMIS creates the appropriate pointers to link the two files when the first report request is processed. Once the data are stored, a RAMIS report request can be written to access the master file to display patient name, date of first visit, length of treatment, and measures of outcome or cost. The formatting of this hypothetical report might undergo a few changes and then be routinely generated for every patient and routed to the therapist periodically.

RAMIS possesses all of the features of a DBMS listed previously. When selecting a DBMS, the manner in which a feature is provided, as well as whether that feature is available, should receive due consideration. Some of the ways RAMIS proved most helpful in creating the psychotherapy research ODMS were (1) the addition of one command word to convert a report request from generating tabular output to creating an external file of data, (2) the apparently unlimited ways of formatting reports, (3) the facilities for executing saved RAMIS routines that interact with the person at the terminal, (4) the use of one standard procedure for describing all types of files, (5) the free-form input of English-like state- ments to data entry and retrieval routines, (6) an interactive procedure for locating or changing single data elements, which facilitated running system tests, (7) the virtual linkage of files that brought different types of data together into one file for data retrieval, and (8) the prompt assistance received via the hot line service.

Naturally, we encountered some difficulties with RAMIS during the creation of our ODMS. One basic problem was solved by using the conditional input format feature to convert our case-record type of data to the transaction-based form of input that RAMIS can process. This solution simply converts the sequence of conditional values in one case record to a sequence of transactions (e.g., the test-item responses that are all linked to the same data element at a higher level in the hierarchy). However, more efficient methods of processing case-type record should be devised. Another problem involved designing the file structure so that specific items on the outcome measures need not be linked hierarchically to only one subscale. The advantage of permitting multiple linkages is to increase the variety of outcome measures that the ODMS can accommodate. The RAMIS solution available at that time, "grouped levels," was not as workable as a new feature RAMIS introduced later, multiple-level linkages of files through the use of concatenated keys. Future revisions of the file design of this ODMS should incorporate the latter solution. Of course, there were, and ever will be, "bugs" in the DBMS. While our application developed more slowly due to bugs, RAMIS staff members did their best to fix the bugs or to assist us in avoiding them.

The CMS [International Business Machines (IBM), 1976] interactive operating system provided an excellent environment for RAMIS; it even outperformed RAMIS at certain tasks. For example, automated data entry was better achieved by creating several CMS executive routines to regulate each step. These routines control the sequence of data processing, interact with an operator who monitors each run, and execute the RAMIS routines. Their capabilities include creating or erasing CMS files, calling RAMIS routines, editing CMS files, printing out messages to the operator, and prompting the operator for information. Entering the one-word name of a CMS routine initiates the execution of all of the specified steps.

By way of illustration, one CMS routine, called Datain, performs all data entry operations for cardimage data. It interacts with the operator to gather information for the data processing run, reports what happens during the run, and supplies the choices for selecting subsequent data processing steps. Datain initiates the execution of RAMIS executive routines, which then perform several data entry steps. The automatic sequencing and initiation of all data entry steps by Datain spares the operator from hav- 
ing to remember this sequence each time new data are added to the data base.

Another useful feature of CMS was its support for the automation of file editing. Editing commands such as "find," "change," "bottom," and so on, can be stacked and executed by a CMS executive routine. For example, the card-image data for each scale are placed in separate CMS files. Each file is automatically column edited to associate the proper item key with each response, before the RAMIS routine is activated to enter these data in the data base.

\section{THE DESIGN OF THE ODMS}

Our application of the available computing technology gave birth to an ODMS for managing outcome data collected from a wide variety of psychological scales, hence the name, Scaling Information Management System (SIMS). The SIMS is presently designed to store item responses and subscale scores for four scales, administered to each patient before and after psychotherapy and on two follow-up occasions. Scales data are linked to a variety of patient demographics and specifics of the therapeutic encounter, both of which are stored in a separate RAMIS file connected by virtual linkage to the scales data file. All data can be entered at a remote terminal and saved in a CMS file or converted to a CMS file from card-image data or other types of files. An operator with minimal knowledge of CMS or RAMIS can perform all data entry at a terminal in from $30 \mathrm{~min}$ to several hours, depending on system response times and the occurrence of data coding errors. Personnel who will access the data base take a 3-day RAMIS course in formulating report requests and designing files.

Table 1 depicts the sequence of operations performed by the SIMS. These operations are generic to any ODMS and are so labeled, borrowing from Broskowski and Attkinson (1981). Research is being conducted on the real-world events associated with the outcomes of brief psychotherapy for treating the stress response syndrome. Data are to be generated employing four psychological scales. Completed forms are collected from patients. These data are coded for entry into temporary RAMIS files. Patient status reports are routinely produced by retrieving data from the temporary files. Permanent storage follows. Subsequent retrievals can be made to update the standardized subscale scores as the patient population grows, to test hypotheses, to ascertain the outcome status of a patient, or to check the completeness of a patient's data.

The RAMIS data entry and report-generating routines compose the bulk of the SIMS. Their execution is controlled by 12 CMS routines, each of which is activated by entering one command word at the
Table 1

Processing of Psychotherapy Research Data

Data Processing Step $\quad$ Actions Taken

Selection of Real-World Events Outcomes of brief psychotherapy provided to patients with stress response syndrome.

Definition of Data Elements

Selected patient characteristics and four measures of the outcome of treatment, which may need revision.

Collection

Patient completes forms before, upon completion of, and twice following treatment.

Coding and Temporary Storage Forms coded onto cards; cards converted to temporary system files as raw data are edited and transformed; checks made for errors.

Routine Retrieval Patient status reports generated for each rating occasion.

Permanent Storage Data transferred from temporary to permanent files.

Data Access

Retrieval/Coding/Storage Normative groups analysis updated and the revised standard scale scores reentered.

Spontaneous Retrieval

On-line report requests initiated at any time to support research and clinical decision making.

terminal. The CMS routines also interact with an operator to collect run-specific information, execute other CMS routines that perform file-editing tasks, or call RAMIS executive routines, which also may interact with the operator. The RAMIS data entry routines read CMS files containing new data and store them in RAMIS files for routine reporting purposes. Later, other data entry routines transfer their data to archival RAMIS files. The reportgenerating routines supply the operator with information about the status of the data and produce copies of the patient status report for filing in the case records. All data entry is performed interactively in real-time. While this version of the SIMS does not contain any user-written computer programs, such programs could be developed to replace steps now performed by RAMIS or CMS, as well as to add new steps.

The SIMS also consists of various data processing protocols. One such protocol, or prescription for interacting with the data base, stipulates that, in general, the CMS routines controlling data entry should be executed in alphabetical order. A protocol for accessing data stipulates that a RAMIS "if" selector should be added to trial report requests to curtail the amount of data sampled, thereby conserving resources, until the output meets all of the user's specifications. Other protocols relate to input data 
formats, variable transformations frequently added to report requests, and steps related to error detection and correction.

The SIMS possesses a modular design, so as to simplify the addition or revision of a scale or input data stream. For example, within the CMS routine Datain, data for each scale are entered separately into the RAMIS files, and the card-image data do not need to be presorted by scale. Thus, routines for processing new scales can be added at any time, without affecting the card-reading step. Presently, a second type of input data, a batch-processing diskbased file, is handled by a separate set of CMS routines. Thus, the SIMS contains an example of how other types of data input streams can be incorporated into its system.

\section{OPERATING THE SIMS}

The purpose of this section is to convey a sense of the skills needed to make use of the SIMS and to further illustrate some of the SIMS features. Some familiarity with on-line data processing is assumed.

The different levels of involvement with the SIMS require three different levels of training. Personnel performing only data entry need the least amount of special training. They should learn how to enter and exit from the CMS environment and how to handle card data and become familiar with the error-detection features in the SIMS. The researchers and their assistants (i.e., personnel involved in data retrieval) must learn how to create and edit CMS files and will need the introductory 3-day RAMIS training that covers file design and the use of the report generator. Personnel who would be involved in modifying or tuning the SIMS should take the 5-day RAMIS training, which also covers the data entry procedures. They must also learn to write CMS routines, particularly those that perform file editing.

\section{THE SIMS FILING STRUCTURE}

The SIMS is designed to input and store item responses and subscale scores for four scales that measure the effects of treatment. Each scale is administered to each patient on four occasions: initial evaluation, termination of treatment, 60 days following treatment, and 1 year later. The Symptom Checklist (SCL) (Derogatis, 1977; Lipman, Rickles, Covi, Derogatis, \& Uhlenhuth, 1969) contains 90 questions about level of symptom discomfort that are grouped into 16 subscales. A life-events questionnaire (Horowitz, Schaefer, Hiroto, Wilner, \& Levin, 1977) requests frequency and recency of occurrence of 45 events generally regarded as quite stressful. These events are grouped into two subscales. The Impact of Event Scale (Horowitz, Wilner, \& Alvarez,
1979) uses frequency of occurrence of 16 typical reactions to a stressful event to assess the current level of a patient's emotional trauma. The reactions are divided into two subsets for scoring purposes. Also, three global ratings of the degree of stress reaction are collected from each patient: degree of reaction when a stressful event occurred, current level of reaction, and amount of adjustment. The SIMS also stores patient demographics and details of the patient's stressful event.

Operating the SIMS in CMS requires that all routines and data be stored as CMS files, including the RAMIS system routines and data files, CMS routines, RAMIS report requests, and the input and output data files. RAMIS data base files contain the coded and transformed data. The SIMS employs four such files: "Archives," a permanent file, contains all of the scaling data and the virtual linkage to the patient identifying data; "Patients," a permanent file, contains some patient demographic information, some specifics of the patient's stressful event, and indexes of the amount of scale data gathered thus far; "Reports," a reused file that is similar in design to Archives, contains new data being summarized for the patient status report on a one-time basis; and "Leqsort," a reused file, handles the input of preprocessed data for one scale, the Life Events Questionnaire.

All queries are addressed to the Archives file, since it contains, or is linked to, all data elements. The design of this file is hierarchical and entity- or patientoriented. Refer to Martin (1976, chap. 3) for a discussion of the relationship of file design to generic types of queries. This particular design makes the running of the patient status reports relatively more efficient. The four "true" levels of the hierarchy of data fields descend from patient identification through rating occasion and scale identification to either subscale scores or item responses. This either-or branch to the lowest level is achieved by making the fifth level of the RAMIS file a "grouped level." In other words, the level containing the item keys and responses is not keyed to the grouped level, which contains the subscale scores and a missing value summary; instead, it is linked directly to the third level, scale identification. This file design is more general, because it handles scales for which two or more subscales share the same items. Also, patient identification is linked to a virtual level (i.e., only data pointers are stored in the virtual level; the actual data are stored in the Patients file).

While the use of a virtual level is transparent to anyone accessing the Archives file, the presence of the grouped level affects certain types of report requests. Subscale scores and item responses should not be combined in the same report request, unless multiple passes through the data base are executed 
in different sections of the request (e.g., when using the RAMIS "store" and "run" options). Except for this one restriction, any type of query involving the data elements, transformations of them, or combinations, such as sums or averages, can be addressed to one file, Archives, at any time following the entry of the data.

\section{Data Entry}

Twelve CMS executive routines control all data entry and generate the patient status report for each of the four rating occasions. These routines are usually executed in alphabetical order by entering the designated name at the terminal. They print out reminders at the end of each run as to the next steps to take. If the wrong routine is entered, it may be terminated by pressing the "return" key after the first pause.

The flow of data entry is outlined in Table 2. Data coding and initial entry are followed by generation of the patient status reports and then archival storage. Errorful data may be discovered during the data entry or report-generation phases. When errors occur, either they are reported during execution of the CMS routine or the operator is told to list a special error report file. At either time all of a patient's new data may have to be deleted from the temporary file to eliminate the error. The corresponding card data must then be segregated and the errors corrected before submission on a subsequent run. Following this procedure assures obtaining more accurate status reports and the permanent storage of highly reliable data for future access.

The entry of data from two rating occasions for one patient, depicted in Figure 1, illustrates many of the SIMS data entry features. The CMS executive routine is called Datain. The operator types in this name at the terminal and then presses the return key to start the routine. First, the run costs are displayed. Second, a check on available disk space is made. At this point the operator may press "return" if the wrong routine was started. However, in this illustration the operator answers "yes" to this question and to the one regarding the readiness of the card data. A SIMS-generated note follows that directs the operator's actions. Next, the routine activates a RAMIS report request to check the appropriateness of the card data. All is well, so the operator again responds "yes." The card data are converted to item-keyed data by special CMS editing routines. Then, these new data files are read into the reports file by RAMIS, after RAMIS notes missing values and calculates the subscale scores. A summary report is created by RAMIS to provide feedback to the operator regarding the accepted data items. The operator should note that one SCL form is missing for this patient. The CMS routine now describes how to obtain the listing of errors, if there were any. As there were no run errors, the SCL form must not
Table 2

SIMS Data Entry Procedure

\begin{tabular}{|c|c|}
\hline Stages of Data Entry & Steps Performed \\
\hline Data Input & $\begin{array}{l}\text { Forms data coded onto cards (or card } \\
\text { image); cards read into CMS data file; } \\
\text { CMS executive routines sort and trans- } \\
\text { form data and direct RAMIS to create } \\
\text { a temporary reporting file, as well as } \\
\text { to store patient identifying information } \\
\text { in a permanent patients file. }\end{array}$ \\
\hline Error Screening I & $\begin{array}{l}\text { Transaction errors are logged in error } \\
\text { files for subsequent listing; special } \\
\text { RAMIS reports are displayed to indicate } \\
\text { the amount of data entered for each } \\
\text { patient; operator reviews these reports; } \\
\text { errors may require deletion of all new } \\
\text { data for that patient; errorful patient } \\
\text { data cards segregated for correction and } \\
\text { reentry. }\end{array}$ \\
\hline
\end{tabular}

Report Generation CMS executive routine generates patient status reports for each rating occasion; reports directed to line printer for rapid output.

Error Screening II Operator reviews reports, noting the special message that identifies missing information on each report; once again, occurrence of errors may require deleting new patient data, using a special CMS executive routine.

Data Input and Error

Screening for

Preprocessed Data

CMS executive routine transfers data from on-line batch processing storage to on-line CMS storage; RAMIS executed to sort and enter recoded data in temporary file; errors and missing data problems noted.

Archival Storage Two CMS executive routines transfer card and preprocessed data from temporary files to merge them with permanently stored data.

Back-Up

Card data stored as raw data back-up; CMS executive routine creates RAMIS file back-up at the beginning of next run; periodically, the permanent files are transferred to tape.

have been included in the card data. The operator would then decide whether to delete all of the data for that one occasion and resubmit the card data when the missing SCL report is located or to continue. If the reports are generated, they will indicate that all SCL data are missing for that occasion. Finally, the cost of running Datain can be calculated. In this case, it totaled \$3.81.

Various-sized runs were performed on test data to gauge the optimal number of rating occasions to include for each data entry run. The sample runs produced a rapidly descending curve of cost of entry and storage per occasion. For one occasion entered per run, it cost \$15; for four occasions per run, the cost per occasion was $\$ 4.25$, and for 30 occasions, 71 cents. This rapid decline in costs leveled off for over 40 occasions per run (i.e., 130 occasions would cost 41 cents each). These costs include only comput- 


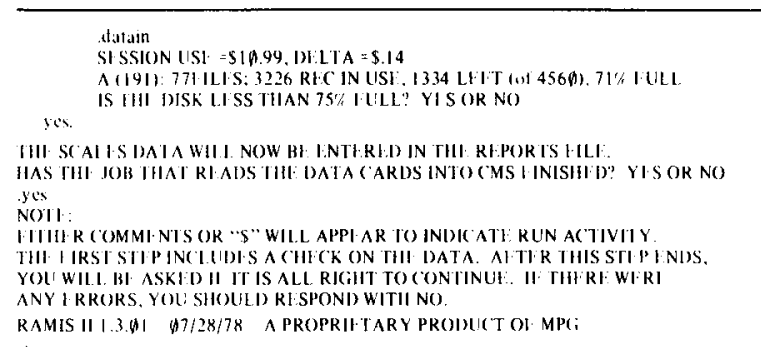

NIIMIBF ROI RI CORIOS IN TABLI:NUMBLR OI RICORDS I:NTIRRII)= NIIMBIER OI RL:CORDS INTI RI:D=

NO IRANSACTION ILRRORS RI [OROI:D

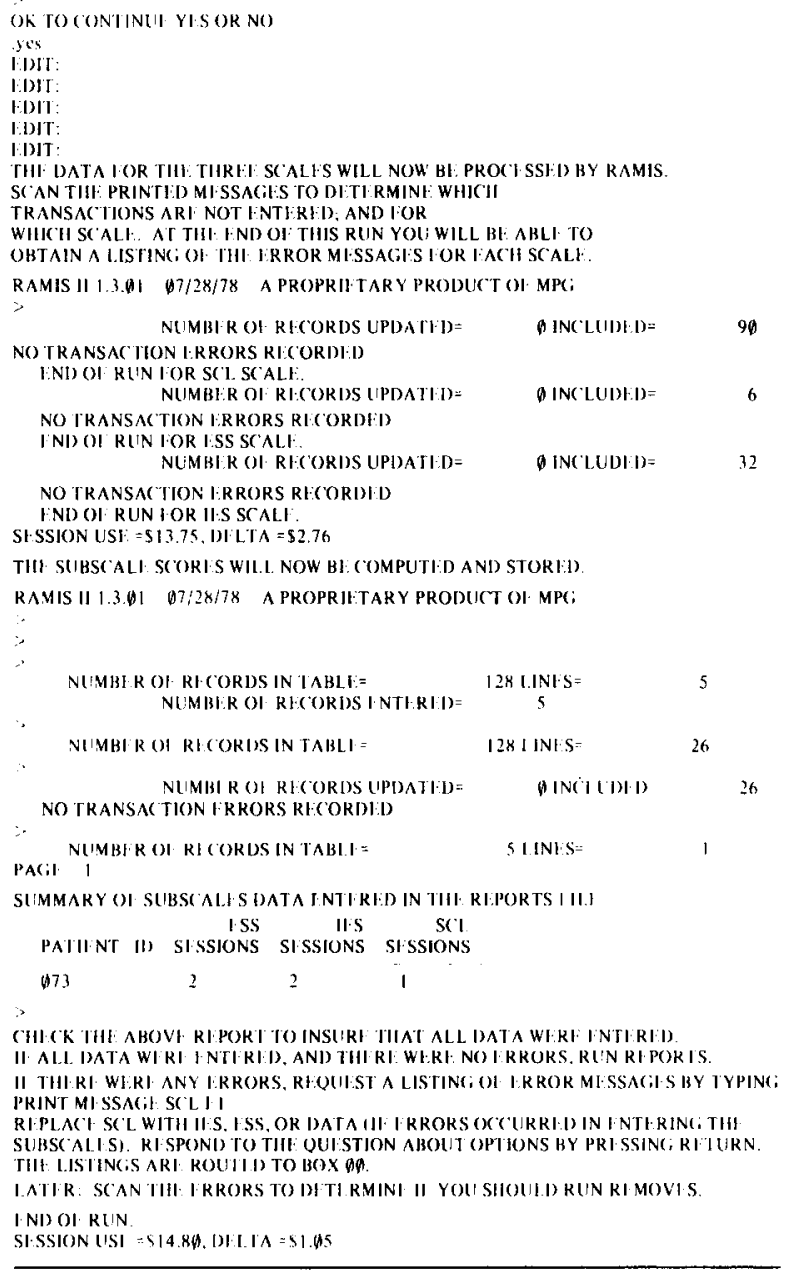

Figure 1. Sample data entry run of two rated occasions for one patient.

ing service costs. Notably, operator's time is not included; ideally, data entry can be performed as a minimal distraction from other data processing duties.

\section{Data Retrieval}

The only data retrievals thus far automated are the reports of each patient's status, supplied after each rating occasion; each report covers three of the outcome measures. Automation of data retrieval should increase, the longer the SIMS is operated, since some spontaneous report requests turn into routine ones over time. For example, one of the data processing staff might complain that help is needed to capture the outcome data in a more timely fashion. A report is constructed that contains a current list of all patients requiring an assessment and which rating occasion is due to be collected. Eventually, this report might be generated during each data entry run and routed to this staff member.

The process of preparing report requests tends to follow this sequence of steps: (1) The question involving the data is stated formally; (2) the question is translated into the language of the DBMS' report generator; (3) a report request is entered at a terminal using the file-editing facilities of the IOS; (4) a selection criterion to limit the sample of data is added to the request until it performs appropriately; (5) the DBMS is activated to execute the request; (6) the results are reviewed and revisions of the query may be made; and (7) Steps 5 and 6 are repeated, or, in some instances, all steps are repeated until a satisfactory report is obtained.

The elements that must be coordinated during this interactive process are the operation of the report generator, the characteristics of the stored data, and the nature of the query. When the researcher reaches Step 6, some thought may have to be devoted to each of these three elements and how they relate to each other before a more appropriate report request can be submitted. Two examples of retrieving data from the SIMS are presented below. Besides illustrating how RAMIS operates, they will highlight the interplay of these three elements.

One question posed by the psychotherapy research staff was "How many patients waited $X$ months following the occurrence of a stressful event before seeking help from their treatment team?" The value of " $X$ " varied from under 3 months to 3-6 months to over 6 months. The variables needed to answer this question included patient information, stress event data, rating occasion number, and test date. The first report request submitted is displayed in Figure 2.

The report is divided into two sections. The "define" section transforms file variables to report variables. Thus, "Xdays" represents the difference in days between the occurrence of the stress event and the evaluation of the patient for therapy, as calculated by a special RAMIS function, "MDY." "Xmon" converts Xdays to the three periods. The "table" section contains the report-generating commands. The verb, "sum," plus a special variable, "count," produce the count of patients. The sequencer, "across," segregates the counts by period and displays them across the page, horizontally. "NE" and "LE" are abbreviated forms of "does not equal" and "is less than or equal to." The last "if" selector restricts the report to only a few records. 


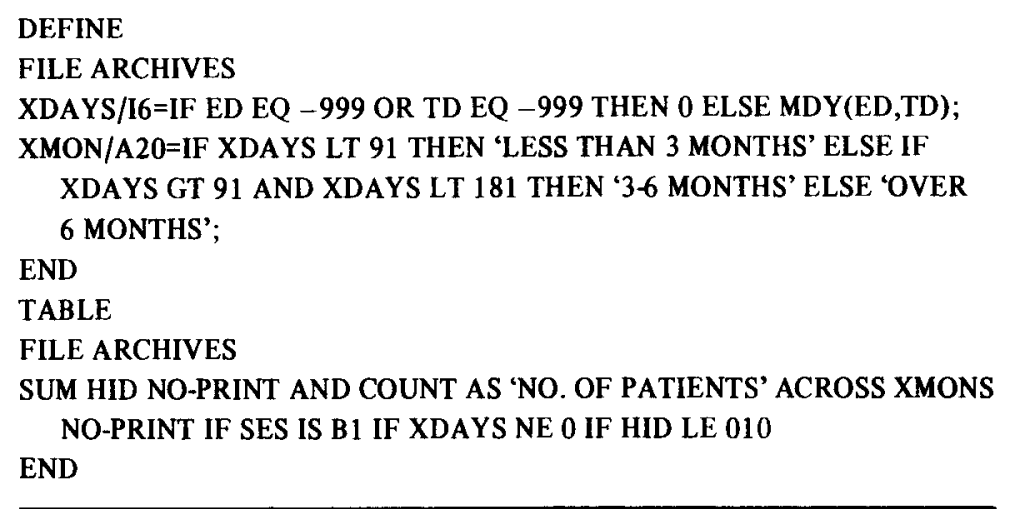

Figure 2. The first report request for calculating how many patients waited each of the three different periods between their stress event and evaluation for therapy.

Upon reviewing the report, several problems were noted. Xmon was misspelled once. The formatting of the output of two variables needed revising. The values of Xmon had to be created in the same order as they were to appear on the report. Finally, a title was added.

After completing the seven-step process noted above and removing the last "if" test, the report depicted in Figure 3 was produced. The results of this report indicate that more than one-third of the patients applied for treatment within 3 months of experiencing a traumatically stressful life event. The total time spent obtaining this report was about $1 \mathrm{~h}$. The computing costs were under $\$ 5$.

The above example illustrates how a summary table can be generated by RAMIS to answer a research question. The same process can be carried a couple of steps further to obtain a statistical analysis of an output data file. The report request in Figure 4 was designed to generate a file of 25 variables for an SPSS correlational analysis. The exploratory question posed was, "How do these variables relate to each other, especially to pre- and posttherapy levels of functioning as measured by selected subscales?"

The appearance of this RAMIS report request is similar to the previous one, except there is no title and a special statement is added at the beginning to link a CMS data file to the RAMIS report generator. The essential difference is the addition of the "save" command, following the sum verb. The contents of the report will be sent to the file, Study1 Data A1, and a summary of the variables and their formats, as stored in the file, will appear at the terminal in place of a tabular report.

Several steps were taken to prepare the output file for the subsequent SPSS analysis. Since sex is coded as alphanumeric in the Archives file, a define statement was created to convert the alphanumeric coding to numeric for SPSS. Missing values are established for the correlation analysis by looking them up in the SIMS manual (-999 for days elapsed between stressful event and evaluation for therapy, 9 for sex). The use of Save, rather than "Savb," produces an output file in EBCDIC, vs. BCD, coding;

\begin{tabular}{|c|c|}
\hline \multicolumn{2}{|l|}{ FILE ARCHIVES } \\
\hline \multicolumn{2}{|c|}{$\begin{array}{c}\text { VIRTUAL BINS REQUESTED }=0 \text { ALLOCATED }=2 \\
\text { NUMBER OF RECORDS IN TABLE }=38 \text { LINES }=1\end{array}$} \\
\hline \multicolumn{2}{|c|}{$\begin{array}{l}\text { NUMBER OF PATIENTS WHO ENTERED C.S.N. } \\
\text { FOR THREE TIME PERIODS SINCE STRESS EVENT }\end{array}$} \\
\hline BEFORE 3 MONTHS & FROM 3-6 MONTHS \\
\hline $\begin{array}{l}16 \\
> \\
\text { R; } \mathrm{T}=1.56 / 2.73 \quad 23: 08: \\
\text { SESSION USE }=\$ 2.96,\end{array}$ & $\mathrm{LTA}=\$ .53$ \\
\hline
\end{tabular}

Figure 3. The final report of patients' waiting periods prior to seeking therapy. 


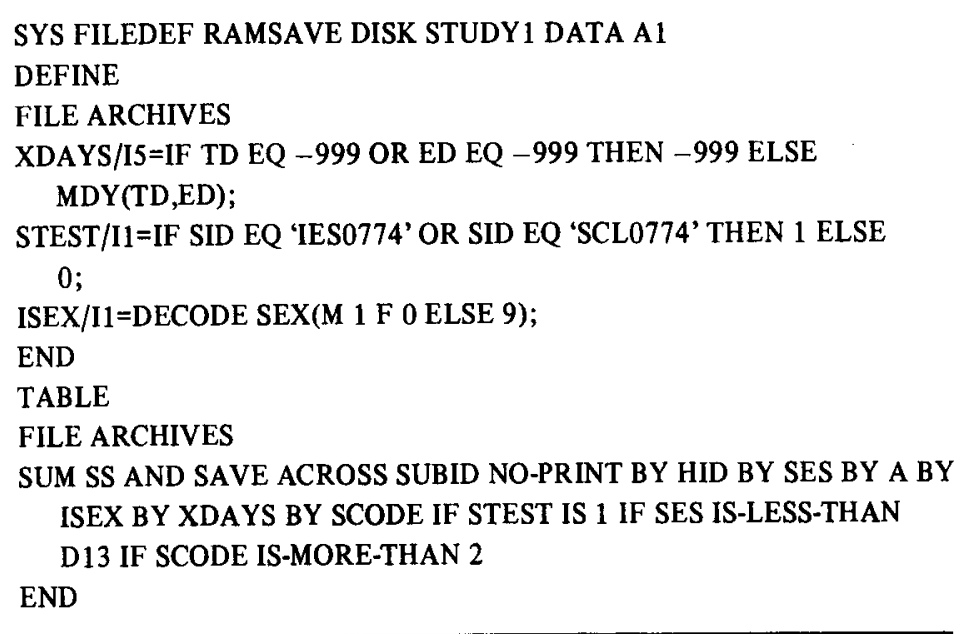

Figure 4. Report request that generates a data file for the correlational study of 25 variables.

EBCDIC coding is easily read by the user, thus facilitating error detection.

After Study1 Data A1 was created, another SPSS card-image file defining the correlation analysis was submitted as an on-line job in CMS. The output from this analysis was briefly screened at the terminal and then routed to the line printer. Some problems with the results were noted that led to the correction of some of the data in the RAMIS Archival file. All of the above steps had to be repeated to obtain the proper correlations. The initial time and costs were less than $2 \mathrm{~h}$ and $\$ 15$. Probably more significant were the ready discovery and correction of the errors in the data base and the ease of editing and reexecuting files in CMS.

\section{ALTERNATIVE SIMS APPLICATIONS}

The SIMS was created to be transportable to other settings. Since no two settings are likely to be served with exactly the same product, we describe below three ways the SIMS may prove helpful to other researchers. Hopefully, these three suggestions hint at a range of uses for the SIMS.

\section{Implementing the SIMS Today}

Setting up another SIMS at a similar site, given the availability of the hardware/software requirements noted in the last section of this report, might proceed as follows. After the programmer has been selected and trained in CMS and RAMIS and has studied the SIMS routines, the researcher would supply the list of scales, the sources of the data, and the data formats. If preprocessed data are to be supplied via batch-processing disk storage, then the routines that process the life-events questionnaire data may be used; otherwise, these routines can be omitted. The programmer would revise the RAMIS file structure, the input routines that convert raw data to itemkeyed data, and the RAMIS routines that enter the file structures in the data base. Most of the revisions could be accomplished quickly, employing the CMS text editor.

Meanwhile, the researcher would prepare the formats for any routine reports that are needed. Selected test data would be supplied to the programmer. After the routines are coded into CMS, a trial run would be conducted on the test data. No doubt, local IOS conventions would dictate minor alterations in the SIMS procedures at this point. The researcher should also select the data processing person, who will operate the SIMS, in time to have this person participate in the test runs. Special runs revising the format of the routine reports will be needed, too.

Taking an optimistic view, the special training of staff might require 2-4 weeks, the set up of the SIMS another 4-6 weeks, and the debugging of actual data processing up to 2 months more. Perhaps, an application employing the SIMS in a similar situation might take as few as 14 weeks to implement.

\section{Borrowing from the SIMS}

Researchers operating in settings quite different from ours may find it useful to borrow pieces from the SIMS. The scenario that follows was developed to illustrate how such borrowing might occur.

Suppose that a grant has been obtained to conduct three field surveys over a 2 -year period in four different regions of the country. The survey instrument must be developed and pilot tested during the first 6 months. The data analyses will be performed by SPSS, so the SIR (Robinson, Anderson, Cohen, Gazdzik, Karpel, Miller, \& Stein, 1980) 
DBMS is chosen. SIR will be operated in TSO (IBM, 1978) on an IBM main-frame computer.

The principal investigator, upon obtaining a copy of the SIMS, is helped as follows. Since responses will be collected from numerous people on three occasions, the file structure for storing data will resemble the SIMS file structure. The sequence of data entry steps proves similar: multiple streams of data that require editing and item keying for instrument development. Thus, time is saved when developing the data entry system for SIR. Since TSO does not perform column overlays when merging files, as does CMS, it is readily determined that a PL/I program must be written to perform the item keying translations. The presence of report summaries in the SIMS data entry routines, which produce immediate feedback on the status of the data accepted by the DBMS, suggest to this investigator some similar reports that SIR can provide. A sorting routine is available, so the timing of sort operations carried out by the SIMS is mimicked by this system to accomplish similar data entry efficiencies.

These cost and time savings, in addition to the early recognition of a need for a user-written program, help this research endeavor along, even though it differs widely from psychotherapy research. The flexibility of the SIMS' design for creating and revising psychological scales makes it possible for the research team to pilot a more reliable survey instrument within the 6 months allowed.

\section{The Future: Microcomputer-Based ODMS}

With such rapid change swirling about us, what will the SIMS of today do for us tomorrow? Tomorrow's computing environment is expected to be heavily populated with microprocessors, as illustrated by a recent Newsweek cover article (Sheils, Cook, Reese, Frons, \& Malamud, 1980). Technological advances in microprocessor chip design are expected to peak in about 5 years. At that time, 16-bit, and even some 32-bit, microprocessor chips will be supporting computing operations now possible only on large main-frame computers. Manufacturers of peripherals-memory storage, communication, and display devices-are pushing their technologies to develop microcomputer-compatible equipment. Tandy Corporation and others are building international distribution networks to deliver "friendly" microcomputer systems to a mass market at affordable prices. The pressure of the rising costs of mainframe computing services will surely accelerate these developments.

Perhaps, 5 years from now, a complete microcomputer system, selling for under $\$ 10,000$, will handle a RAMIS-like DBMS and a CMS-type IOS. The hardware configuration for such a system will include a CPU, video display with graphics capabilities, a fine-quality 100 -cps line printer, sizable hard-disk on-line storage, floppy disk or magnetic bubble memory off-line storage, and communications linkage to other video displays and to main frames operated by centralized computing services.

The present design of the SIMS makes it readily transportable to this computing system of the future. Assuming that a different operating system and DBMS will require a major translation effort, the sequence of data entry operations, the nature of the data transformations, and the interactive query process will still be worth imitating. New routines can be created to generate card-image data on-line as they are keyed in from the original forms. Interactive queries can be rapidly developed and processed, because computer response times are minimal, the video display and line printer operate quite rapidly, and a hard copy is immediately available for others to review. The communications linkages will handle the off-line printing of batched reports or the dumping of output files for statistical analyses at a mainframe computing service center.

No doubt, only pieces of the SIMS will serve other applications. The inherently modular design will make it relatively easy to drop and add routines. For example, the entry of patient demographics occurs separately from the entry of scale data, which occurs separately from the entry of any preprocessed data. Even the routines for entering the various scales permit adding or deleting scales one at a time. Other routine reports can be added during data entry, or the present one can be omitted.

Clearly, other applications of the SIMS could play an important role in handling future data management tasks on a microcomputer system. In fact, microcomputer applications should yield still more optimal ODMSs by providing full and inexpensive access to reliable, relevant, and comprehensive data, at the push of a key, and, perhaps, in response to vocal commands.

\section{THE AVAILABILITY OF THE SIMS}

A copy of the SIMS manual (Green \& Attkisson, Note 2) can be purchased at nominal cost. This 80-page booklet describes the SIMS, lists the data entry steps, and explains the process of retrieving data. The appendices contain the system routines: 15 CMS routines along with 13 RAMIS routines, four file descriptions, four data conversions for some frequently needed variables that are not stored, the missing values table, and the formats for the data that are input to the SIMS.

The SIMS must be operated in CMS, IBM's Conversational Monitor System. CMS manuals can be obtained by writing: IBM Corp., VM/370 Publications, Dept. D58, Bldg. 706-2, P.O. Box 390 , Poughkeepsie, New York 12602. The RAMIS II operating manuals and training schedules can be 
obtained by writing: Mathematica Products Group, P.O. Box 2392 Princeton, New Jersey 08540. Other software needed for immediate operation includes a rapid sorting program and linkages to a batch system for reading cards or disk files into the CMS environment. The hardware items, in addition to an IBM main-frame computer, should include a hard-copy terminal that prints 100 or more columns for communicating with the main frame, and a line or page printer run by the main frame.

\section{REFERENCE NOTES}

1. Karpinski, R. Personal communication, September S, 1980.

2. Green, R. S., \& Attkisson, C. C. SIMS: A scaling information management system for psychotherapy research. Unpublished manuscript, 1979. (Available from C. Clifford Attkisson, Department of Psychiatry, University of California, San Francisco, California 94143.)

\section{REFERENCES}

Attkisson, C. C., Hargreaves, W. A., Horowitz, M. J., \& SORENSEN, J. E. (Eds.). Evaluation of human service programs. New York: Academic Press, 1978.

Broskowski, A., \& Atkisson, C. C. Information systems for health and human services. New York: Human Sciences Press, in press.

Chapman, R. L. The design of management information systems for mental health organizations: $A$ primer (DHEW Publication No. ADM 76-333). Washington, D.C: U.S. Government Printing Office, 1977.

DAvis, G. B. Management information systems: Conceptual foundations, structure, and development. New York: McGrawHill, 1974.

Denogatis, L. R. SCL administration, scoring, and procedures manual-I. Baltimore, Md: Johns Hopkins, 1977.

ENGER, N. L. Management standards for developing information systems. New York: AMACOM, 1976.

Hargreaves, W. A., Attikision, C. C., \& Sorensen, J. E. (Eds.). Resource materials for community mental health program evaluation (DHEW Publication No. ADM 77-328). Washington, D.C: U.S. Government Printing Office, 1977.
Horowitz, M. J., Schaefer, C., Hiroto, D., Wilner, N., \& LEvin, B. Life event questionnaires for measuring presumptive stress. Psychosomatic Medicine, 1977, 39, 413-431.

Horowitz, M. J., Wilner, N., \& Alvarez, W. Impact of event scale: A measure of subjective distress. Psychosomatic Medicine, 1979, 41, 209-218.

International Business Machines. IBM virtual machine facility/370: CMS user's guide (File No. S370-30, Order No. GC20-1819-0). Poughkeepsie, N.Y: Author, 1976.

International Business Machines. OS/VS2 TSO terminal user's guide (Sth ed.; File No. S370-39, Order No. GC28-0645-4). Poughkeepsie, N.Y: Author, 1978.

Johnson, J. H. A practical guide to installing a computer system in a mental health setting. In J. B. Sidowski, J. H. Johnson, \& T. A. Williams (Eds.), Technology in mental health care delivery systems. Norwood, N.J: Ablex, 1980.

Krauss, L. I. Computer-based management information systems. New York: American Management Association, 1970.

Lipuan, R. S., Rickles, K., Covi, L., Derogatis, L. R., \& Uhrenhuth, E. H. Factors of symptom distress. Archives of General Psychiatry, 1969, 21, 328-338.

Martin, J. Principles of data-base management. Englewood Cliffs, N.J: Prentice Hall, 1976.

Martin, J. Computer data base organization (2nd ed.). Englewood Cliffs, N.J: Prentice Hall, 1977.

Mathematica Products Group. RaMIS users manual (rev. ed.). Princeton, N.J: Author, 1977.

Nie, N. H., Hull, C. H., Jenkins, J. G., Steinbrenner, K. \& BENT, D. H. SPSS: Statistical package for the social sciences (2nd ed.). New York: McGraw-Hill, 1975.

Robinson, B. N., Anderson, G. D., Cohen, E., Gazdzik, W. F., Karpel, L. C., Miller, A. H., \& Stein, J. R. SIR users manual: Version 2. Evanston, III: SIR, 1980.

Rosenberg, M. IBM compatible products review. Computer World, July 14, 1980, pp. $1 ; 8 ; 10-11$.

Sheils, M., Cook, W. J., Reese, M., Fron, M., \& Malamud, P. Machines that think. Newsweek, June 30, 1980, pp. S0-56.

Sidowgri, J. B., Johnson, J. H., \& Williams, T. A. (Eds.). Technology in mental health care delivery systems. Norwood, N.J: Ablex, 1980.

Strerel, M. L. Surveying data base management systems. Minimicro systems, 1979, 12, 94-104.

WAskow, I. E., \& PARLoff, M. B.(Eds.). Psychotherapy change measures (DHEW Publication No. ADM 74-120). Washington, D.C: U.S. Government Printing Office, 1974.

Appendix

Glossary of Acronyms

\begin{tabular}{cc}
\hline Type of System & Glossary of Acronyms \\
\hline DBMS & Specific System \\
\hline
\end{tabular}

Data Base Management System: The computer program that performs data storage and retrieval, as directed by user-written, English-like routines.

\section{IOS}

Interactive Operating System: A computer-specific program that interfaces users to computers so they can perform real-time (vs. batch mode) computing via remote terminals.

\section{ODMS}

On-Line Data Management System: A set of English-like routines and protocols for storing and accessing data employing a DBMS and an IOS.
Rapid Access Management Information System: A DBMS marketed by Mathematica Products Group, Princeton, New Jersey.

\section{CMS}

Conversational Monitor System: An IOS supplied by IBM for their 370-series main-frame computers that treats each user as a computer operator of a virtual, rather than real, machine.

\section{SIMS}

Scaling Information Management System: An ODMS that stores and accesses data on patients and the outcomes of their psychotherapy, as registered on four scales collected at four points in time. Consists of RAMIS and CMS routines; user-written programs may be added. 